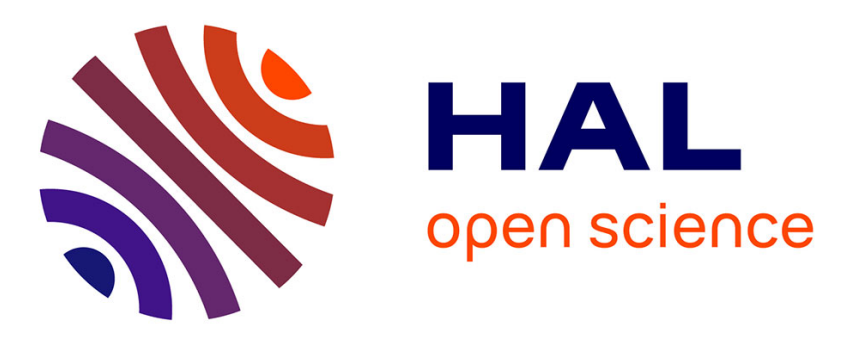

\title{
Energy efficient control derived from homogeneous algorithm - Application to a wind system
}

Elias Tahoumi, C. Evangelista, Franck Plestan, Malek Ghanes, Jean-Pierre Barbot, Paul Puleston

\section{- To cite this version:}

Elias Tahoumi, C. Evangelista, Franck Plestan, Malek Ghanes, Jean-Pierre Barbot, et al.. Energy efficient control derived from homogeneous algorithm - Application to a wind system. Control Engineering Practice, 2020, 103, pp.104586. 10.1016/j.conengprac.2020.104586 . hal-02943838

\section{HAL Id: hal-02943838 \\ https://hal.science/hal-02943838}

Submitted on 7 Nov 2021

HAL is a multi-disciplinary open access archive for the deposit and dissemination of scientific research documents, whether they are published or not. The documents may come from teaching and research institutions in France or abroad, or from public or private research centers.
L'archive ouverte pluridisciplinaire HAL, est destinée au dépôt et à la diffusion de documents scientifiques de niveau recherche, publiés ou non, émanant des établissements d'enseignement et de recherche français ou étrangers, des laboratoires publics ou privés. 


\title{
Energy efficient control derived from homogeneous algorithm - Application to a wind system
}

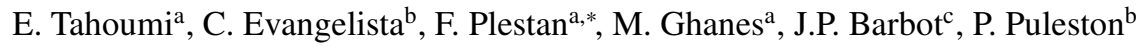 \\ ${ }^{a}$ ECN-LS2N, Nantes, France \\ ${ }^{b}$ LEICI, Faculty of Engineering, UNLP-CONICET, La Plata, Argentina \\ ${ }^{c}$ ENSEA-QUARTZ, Cergy-Pontoise, France
}

\begin{abstract}
A new control methodology derived from higher order sliding mode control (HOSMC) strategy has been recently presented. It ensures high accuracy and requires reduced control effort and chattering; however, only finite time convergence has been proven. In this paper, this controller is revisited and domains of convergence are given for some cases. The second objective is to use this controller to stabilize a twin wind turbine (TWT) system. The performance of the proposed control strategy is evaluated and compared to HOSMC.
\end{abstract}

Keywords: Uncertain nonlinear systems, Higher order sliding mode control, Robust control, Homogeneous control, Wind turbine.

\section{Introduction}

Sliding mode control (SMC) [1],[2] is a very well known robust control method for uncertain nonlinear systems. It is mainly recognized for its robustness against perturbations/uncertainties and high accuracy where these features make it ideal for many applications [3],[4],[5]. However, the main drawback of the standard SMC is the chattering phenomenon i.e. high frequency oscillations which may damage physical components such as actuators and decrease the accuracy with respect to a sampled controller. It is also restricted to systems with input-output relative degree equal to 1 .

Higher order sliding mode control (HOSMC) techniques have been designed to deal with these drawbacks. Typically, they have been designed using homogeneity tools [6], [7], [8] and more recently using a Lyapunov framework [9], [10], [11]. They relieve the restriction on the relative degree and increase the closed-loop system accuracy. Despite that, the chattering reduction is limited due to the existence of a discontinuous control action in the higher derivatives of the sliding variable.

In this paper, the problem under interest is the stabilization of a perturbed chain of integrators with arbitrary degree, retaining the advantages of HOSM control (accuracy and robustness) but with their drawbacks reduced (chattering and high energy consumption). Such attempts have been made for second order systems $[12,13]$. The proposed solution was based on linear and

\footnotetext{
${ }^{*}$ Corresponding author

Email address: franck.plestan@ec-nantes.fr (F. Plestan)
}

Preprint submitted to Control Engineering Practice

June 4, 2020 
twisting controllers: a time-varying parameter allows the controller to balance between a linear controller and the twisting one. The obtained controller has the advantages of the twisting controller [14] (accuracy and robustness) and of the second order linear state feedback (low energy consumption). It was also successfully implemented on an experimental setup in [15]. A solution that generalizes this task to a system of arbitrary order has been introduced in [16] that is based on the homogeneous controller proposed in [17]. The latter is a HOSM controller recognized for its high accuracy and robustness. However, the gains are overestimated resulting in a high energy consuming controller. Controller [16] has the advantages of the homogeneous one in [17] (accuracy and robustness), but with its drawbacks (chattering and high energy consumption) strongly reduced. This is made possible by introducing a parameter on the exponent term of the homogeneous controller [17], this parameter varying between 0 and 1 and depending on the accuracy of the tracking. However, the convergence domain of this controller has not been calculated and only finite time convergence is proven. The first objective of this paper is to formally give the convergence domain of the proposed controller in [16] when the relative degree is equal to 1 and 2 .

The second objective is the design and implementation of a controller for a twin wind turbine system, SEREO [18]. It includes two identical wind turbines mounted on to the same tower. Due to this fact, no additional yaw actuator is required to follow the wind direction; this is done by creating a difference in the drag force of both wind turbines which in turn induces the yaw motion. The TWT belongs to the family of uncertain nonlinear systems and therefore could be difficult to control. Indeed, it is a delicate task to mathematically model its dynamics as well as the perturbations that are acting on it. This makes the proposed controller an ideal tool to achieve the control objectives given its robustness with respect to matching perturbations/uncertainties. It is shown via simulations that the designed control scheme successfully forces the structure to face the wind while keeping optimal energy production. Two aspects of the control are considered

- mechanical: controlling the yaw motion of the structure in order to orient it face the wind by acting on the blade pitch angle of both twin turbines.

- electrical: forcing the direct current of both generators to 0 to limit the ripple effect on the electromagnetic torque and controlling the angular velocities of both generators in order to optimize the electrical power output. This is achieved by acting on the direct and quadrature stator voltages.

Once the system is decoupled, the input-output relative degrees of each decoupled part are 1, 2 and 3. This makes the proposed control strategy ideal given that the proposed controller has no restriction on the relative degree.

The paper is organized as follows. Section 2 defined the TWT model. Section 3 details the control objective. Section 4 details the controller and the convergence domain for some cases. Section 5 the proposed controllers are implemented via simulation and their performances are evaluated.

\section{Model of the SEREO structure}

The SEREO Twin Wind Turbine (TWT) (see Figure 1 and 2) is made of two identical wind turbines mounted on a tower. The rotation face the wind needs no yaw driving motor. The yaw motion is generated by creating a drag forces difference between both wind turbines, thanks to an 


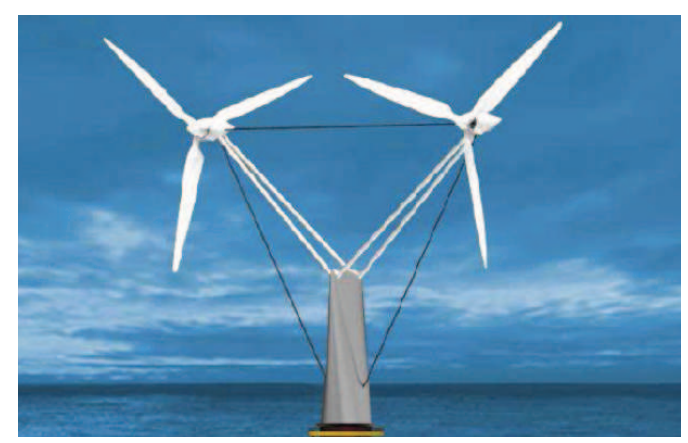

Figure 1: The SEREO structure [18].

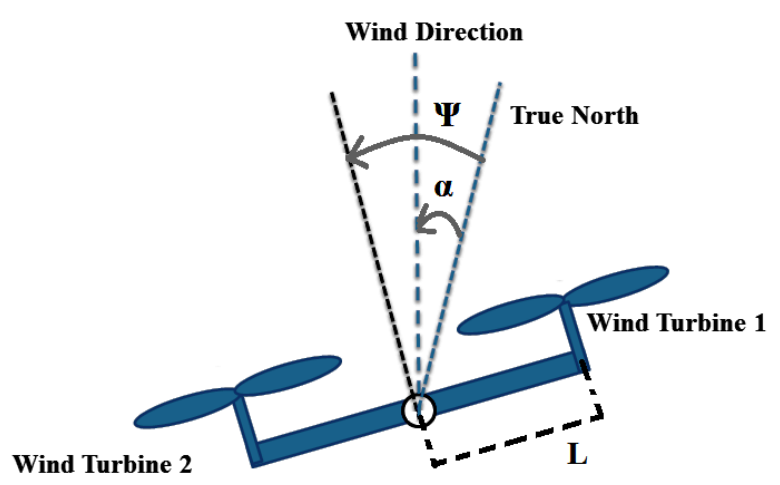

Figure 2: Simplified model (top view).

action on the blade pitch angles. The difference between the blade pitch angles is made around the blade pitch optimal angle. The SEREO model reads as ( $x$ being the state vector and $u$ the control input vector)[5]

$$
\dot{x}=f(x)+g(x) \cdot u
$$

with

$$
\begin{aligned}
& x=\left[\begin{array}{llllllllll}
\beta_{1} & \beta_{2} & \psi & \dot{\psi} & i_{d 1} & i_{q 1} & \Omega_{1} & i_{d 2} & i_{q 2} & \Omega_{2}
\end{array}\right]^{T}, \\
& u=\left[\begin{array}{llllll}
\Delta \beta_{1} & \Delta \beta_{2} & V_{d 1} & V_{q 1} & V_{d 2} & V_{q 2}
\end{array}\right]^{T}
\end{aligned}
$$


and

$$
\begin{aligned}
& f(x)=\left[\begin{array}{c}
\frac{1}{T_{\beta 1}}\left(\beta_{1}^{o p t}-\beta_{1}\right) \\
\frac{1}{T_{\beta 2}}\left(\beta_{2}^{o p t}-\beta_{2}\right) \\
\dot{\psi} \\
\frac{1}{K_{r}}\left(-D_{r} \dot{\psi}+\left(F_{d, 1}-F_{d, 2}\right) L\right) \\
\frac{-R_{s}}{L_{d}} i_{\mathrm{d} 1}+\frac{p L_{q}}{L_{d}} i_{\mathrm{q} 1} \Omega_{1} \\
\frac{-R_{s}}{L_{q}} i_{\mathrm{q} 1}-\frac{p L_{d}}{L_{q}} i_{\mathrm{d} 1} \Omega_{1}-\frac{p \phi_{f}}{L_{q}} \Omega_{1} \\
\frac{p \phi_{f}}{J} i_{\mathrm{q} 1}-\frac{p\left(L_{d}-L_{q}\right)}{J} i_{\mathrm{d} 1} i_{\mathrm{q} 1}-\frac{f_{v}}{J} \Omega_{1} \\
\frac{-R_{s}}{L_{d}} i_{\mathrm{d} 2}+\frac{p L_{q}}{L_{d}} i_{\mathrm{q} 2} \Omega_{2} \\
\frac{-R_{s}}{L_{q}} i_{\mathrm{q} 2}-\frac{p L_{d}}{L_{q}} i_{\mathrm{d} 2} \Omega_{2}-\frac{p \phi_{f}}{L_{q}} \Omega_{2} \\
\left.\Omega_{1}, \psi\right) \\
\frac{p \phi_{f}}{J} i_{\mathrm{q} 2}-\frac{p\left(L_{d}-L_{q}\right)}{J} i_{\mathrm{d} 2} i_{\mathrm{q} 2}-\frac{f_{v}}{J} \Omega_{2}
\end{array}\right] \\
& g(x)=\left[\begin{array}{cccccc}
\frac{1}{T_{\beta 1}} & 0 & 0 & 0 & 0 & 0 \\
0 & \frac{1}{T_{\beta 2}} & 0 & 0 & 0 & 0 \\
0 & 0 & 0 & 0 & 0 & 0 \\
0 & 0 & 0 & 0 & 0 & 0 \\
0 & 0 & \frac{1}{L_{d}} & 0 & 0 & 0 \\
0 & 0 & 0 & \frac{1}{L_{q}} & 0 & 0 \\
0 & 0 & 0 & 0 & 0 & 0 \\
0 & 0 & 0 & 0 & \frac{1}{L_{d}} & 0 \\
0 & 0 & 0 & 0 & 0 & \frac{1}{L_{q}} \\
0 & 0 & 0 & 0 & 0 & 0
\end{array}\right]
\end{aligned}
$$

where

- the first two equations are the pitch angle dynamics with $\beta_{i}\left(^{\circ}\right)(i \in\{1,2\})$ the pitch angles, $\beta_{i}^{\text {opt }}$ the optimal pitch angle giving maximum power output and considered constant and 
$T_{\beta i}$ the time constant of the blades actuation systems. $\Delta \beta_{i}$ is the control input acting on the structure's rotation.

- the third and fourth equations are yaw-dynamics where $K_{r}\left(\mathrm{~kg} \cdot \mathrm{m}^{2}\right)$ and $D_{r}(\mathrm{~N} . \mathrm{m} /(\mathrm{rad} / \mathrm{s}))$ are respectively the moment of inertia and the coefficient of friction associated to the yaw motion. $L$ represents the distance between each hub and the tower axis (Figure 2). $F_{d, i}(N)$ are the drag forces given as

$$
F_{d, i}=\frac{1}{2} \rho \pi(R V \cos (\psi-\alpha))^{2} C_{d, i}
$$

with $R(\mathrm{~m})$ the wind turbines' blade radius, $\rho\left(\mathrm{kg} / \mathrm{m}^{3}\right)$ the density of air, $\mathrm{Vm} / \mathrm{s}$ the velocity of the wind, $\alpha\left(^{\circ}\right)$ the angle between the wind direction and the true north, $\psi\left(^{\circ}\right)$ the angle between the true north and the orientation of the TWT given by the perpendicular to the arm joining the two turbines (see Figure 2) and $C_{d, i}$ the drag force coefficient which is a nonlinear function of the pitch angle and the tip-speed ratio (TSR), $\lambda_{i},[19]$ which is defined as

$$
\lambda_{i}=\frac{\Omega_{i}}{V \cos (\psi-\alpha)} R .
$$

- The last six equations represent the electrical model with $\Omega_{i}(\mathrm{rad} / \mathrm{s})$ the rotational speed, $\Gamma_{a i}(N . m)$ the aerodynamic torque, $i_{d i}, i_{q i}(A), V_{d i}, V_{q i}(V)$ respectively the direct/quadrature currents and voltages, $L_{d}, L_{q}(H)$ the $d q$-axis inductances, $R_{s}(\Omega)$ the stator winding resistance, $p$ the number of pole pairs, $J\left(\mathrm{~kg} \mathrm{~m}^{2}\right)$ the total moment of inertia, $\phi_{f}(\mathrm{~Wb})$ the flux of the permanent-magnet and $f_{v}$ the coefficient of friction. Note that the considered generator for each twin turbine is a permanent magnet synchronous generator (PMSG).

As it will be presented in Section $3, \Omega_{1}$ and $\Omega_{2}$ are forced to follow the reference $\Omega^{*}$. In this case, considering that $\Omega_{1} \approx \Omega_{2}$, then

$$
\lambda_{1} \approx \lambda_{2} \approx \lambda \text { and } \beta_{1}^{o p t} \approx \beta_{2}^{o p t} \approx \beta^{o p t} .
$$

Taking into account that the rotation is obtained by the difference between $F_{d, 1}$ and $F_{d, 2}$, a symmetric behavior is stated such that $\Delta \beta_{1}=-\Delta \beta_{2}=\Delta \beta$. A new control input $\bar{u}$ is defined as

$$
\bar{u}=\left[\begin{array}{ccccc}
\Delta \beta & V_{d 1} & V_{q 1} & V_{d 2} & V_{q 2}
\end{array}\right]^{T}
$$

In fact, the control inputs $u$ and $\bar{u}$ are $6 \times 1$ and $5 \times 1$ vectors respectively and are linearly linked as follows

$$
u=\left[\begin{array}{ccccc}
1 & 0 & 0 & 0 & 0 \\
-1 & 0 & 0 & 0 & 0 \\
0 & 1 & 0 & 0 & 0 \\
0 & 0 & 1 & 0 & 0 \\
0 & 0 & 0 & 1 & 0 \\
0 & 0 & 0 & 0 & 1
\end{array}\right] \cdot \bar{u} .
$$

\section{Control Problem}

The primary control objective is to ensure that the SEREO Twin Wind Turbines has optimum power production. This objective is achieved by orienting the structure facing the wind, while 
each turbine must operate at its maximum conversion efficiency. Therefore, a yaw angle control is required to maintain the nacelle at optimal orientation [20], together with a MPPT control setup acting on the turbines rotational speeds. Hence, three control problems are considered

- forcing the TWT face the wind, i.e. $\psi-\alpha=0$; when it is not the case, the pitch angle of each wind turbine blade is changed producing a difference between the drag forces $F_{d 1}$ and $F_{d 2}$. A yawing torque is then induced forcing the rotating motion;

- controlling the wind turbines angular velocities as to optimize electrical power. This is performed by maintaining the TSRs at their optimal values $\lambda^{\text {opt }}$, for given blades pitch angles. Hence, the rotational speeds of both wind turbines are controlled to track the reference

$$
\Omega^{*}=\frac{V \cos (\psi-\alpha)}{R} \lambda^{o p t}
$$

- forcing both generators direct currents $i_{d, 1}$ and $i_{d, 2}$ to 0 to avoid electromagnetic torques ripple effects, which may increase the fatigue loads in the mechanical shaft of the wind turbine and affect power production.

\section{New control methodolody}

As seen in Section 2, the TWT is a system with nonlinear dynamics. It is not a trivial task to identify many of its generators' parameters such as the stator winding resistances $R_{S}$ and the inductances $L_{q}$. These parametric uncertainties strongly affect the dynamics of the system. Then, in order to achieve the control objective defined in the previous section, the chosen control strategy is based on sliding mode control (SMC). Indeed, SMC is known for its robustness to matching perturbations/uncertainties. Other advantages of SMC are finite time convergence and its relative simplicity for application. A first attempt to control the TWT has been presented in [5]. A first order sliding mode controller [1] has been used to stabilize the system. However, it suffers from the chattering phenomena which may degrade the accuracy of the tracking and renders the controller high energy consuming. Chattering also induces oscillations to the electromagnetic torque which may increase the fatigue loads in the mechanical shaft of the wind turbine and affect power production.

Then, a solution consists in using higher order sliding mode control (HOSMC) as the one presented in [17], but the chattering reduction is limited since the sign function is still used in the control (main cause of chattering in the case where a digital controller is used). A way to reduce the chattering and the energy consumption even further has been presented in [16]. It introduces a parameter $\mu$ to the exponent term of the controller presented in [17]. However, the convergence domain is not determined. In the sequel, the controller from [16] is recalled. Then,

- the convergence domain of the closed loop is calculated for some cases;

- the design of a controller for the TWT is based on this controller that achieves the control objectives defined in the previous section. 


\subsection{Some recalls}

As previously mentioned, the purpose of SMC is to propose a control law that forces the output of the system to track a reference trajectory. Consider the following system ${ }^{1}$

$$
\begin{aligned}
\dot{\xi} & =f(\xi, t)+g(\xi, t) u \\
s & =s(\xi, t)
\end{aligned}
$$

with $\xi \in \Xi \subset \mathbb{R}^{n}$ the state vector ( $\Xi$ being an open bounded subset of $\mathbb{R}^{n}$ and $n$ being the state dimension), $f$ and $g$ sufficiently differentiable uncertain functions, $u \in \mathcal{U} \subset \mathbb{R}$ the control input ( $\mathcal{U}$ being a bounded open subset of $\mathbb{R}$ ) and $s$ the sliding variable. Assume that

A1. The relative degree of (11), $\rho \geq 1 \in \mathbb{N}$ (where $\mathbb{N}$ is the set of natural numbers), is constant and known, i.e.

$$
s^{(\rho)}=a(\xi, t)+b(\xi, t) u
$$

A2. The internal dynamics of the system are bounded.

A3. $a(\xi, t)$ and $b(\xi, t)$ are unknown but bounded functions such that $\forall \xi \in \Xi$ and $t \in \mathbb{R}^{+}$

$$
|a(\xi, t)| \leq a_{M}, \quad 0<b_{m} \leq b(\xi, t) \leq b_{M}
$$

with $a_{M}, b_{m}, b_{M} \in \mathbb{R}^{+}$.

Remark 1. If the system is at least Lyapunov stable, it is enough to verify that the initial state is bounded because in this case, the state stays in the bounded space $\Xi$.

Under these assumptions, $s$ satisfies the following differential equations

$$
\begin{aligned}
& \dot{z}_{i}=z_{i+1}, \quad i=1, \cdots, \rho-1 \\
& \dot{z}_{\rho}=a(\xi, t)+b(\xi, t) u
\end{aligned}
$$

with $z=\left[z_{1}, \cdots, z_{\rho}\right]^{T}=\left[s, \cdots, s^{(\rho-1)}\right]^{T} \in \mathcal{Z} \subset \mathbb{R}^{\rho}$. The control objective is achieved when $z$ evolves around a vicinity of the origin in finite time regardless of perturbations/uncertainties. In the sequel, the controller introduced in [16] and inspired by [17] is recalled. This latter is a homogeneous controller which allows the establishment of a $\rho^{\text {th }}$ order sliding mode i.e. $z$ reaches zero in finite time. However, as recalled previously, this controller is high energy consuming from a control effort point of view (high gain value). It also engenders the chattering phenomenon caused by the sign function used in the control. This effect is further amplified by the gain overestimation.

The controller in [16] deals with these drawbacks by introducing a parameter $\mu$. This latter is time-varying and gives rise to an efficient trade-off between accuracy and energy consumption.

\footnotetext{
${ }^{1}$ For a sake of clarity, the recalls and control methodology are presented in SISO context.
} 
In the next section, this controller is formalized and the convergence domain is evaluated when $\rho$ equals 1 and 2 .

The controller proposed in [16] reads as 23

$$
\begin{gathered}
u=-k_{\rho}\left\lceil\sigma_{\rho}\right\rfloor^{\mu}, \\
\sigma_{i}=\left\lceil z_{i}\right\rfloor^{\frac{r_{1}}{r_{i}}}+k_{i-1}^{\frac{r_{1}}{r_{i}}} \sigma_{i-1}
\end{gathered}
$$

with $i \in\{2, \cdots, \rho\}, \sigma_{1}=z_{1}$ and $r=\left(r_{1}, \cdots, r_{\rho}\right)=(\rho, \rho-1, \cdots, 1)$. The parameter $\mu$ reads as the following variation law ${ }^{4}$

$$
\mu=\max \left(-\beta \sum_{i=1}^{\rho} \frac{\left|z_{i}\right|}{\left|z_{i}\right|+\varepsilon_{z_{i}}}+1,0\right)
$$

with $\varepsilon_{z_{i}}>0(i \in\{1, \cdots, \rho\})$ and $\beta>1$ tuned by the user. It means that $0 \leq \mu \leq 1$. In fact, when the trajectory of the system is far from the origin, $\mu=0$ and the standard SMC [1] is obtained for $\rho=1$ whereas for $\rho>1$, controller [17] is obtained. Hence, for $\rho=1$, the controller gain $k_{1}$ is tuned as for the standard SMC

$$
b_{m} k_{1}-a_{M}>0
$$

in order to ensure trajectory convergence to a vicinity of the origin. Analogously, for $\rho \geq 2$, $\left(k_{1}, \cdots, k_{\rho}\right)$ are the controller gains tuned as for controller [17] with $\left(k_{2}, \cdots, k_{\rho}\right)$ parameterized with respect to $k_{1}$ as follows

$$
\begin{gathered}
k_{i}=\gamma_{i-1} k_{1}^{\frac{\rho}{\rho-(i-1)}}, \quad \forall i=2, \cdots, \rho-1 \\
k_{\rho} \geq \frac{\gamma_{\rho-1} k_{1}^{\rho}+a_{M}}{b_{m}}
\end{gathered}
$$

where $\gamma_{i}, i \in\{1, \cdots, \rho-1\}$ are suitable positive constants. They are derived from Lyapunov based stability analysis [17]. In fact, they are calculated by evaluating homogeneous functions and numerically finding their maxima on a homogeneous sphere. The proposed values of $\gamma_{i}, i \in$ $\{1, \cdots, \rho-1\}$ for $\rho=2,3,4$ in [17] are given in Table 1 .

\begin{tabular}{l||l}
$\rho$ & \multicolumn{1}{|c}{ Parameters } \\
\hline 2 & $\gamma_{1}=1.26$ \\
3 & $\gamma_{2}=9.62, \gamma_{1}=1.5$ \\
4 & $\gamma_{3}=739.5, \gamma_{2}=8.1, \gamma_{1}=2$
\end{tabular}

Table 1: Values of parameters $\gamma_{i}(i=1, \cdots, \rho-1)$.

On the other hand, when the trajectory of the system is close to the origin is close to the origin, $\mu$ evolves between 0 and 1 . This fact will be discussed in more detail in the sequel.

\footnotetext{
${ }^{2}\left\lceil\sigma_{\rho}\right\rfloor^{\mu}=\left|\sigma_{\rho}\right|^{\mu} \operatorname{sign}\left(\sigma_{\rho}\right)$.

${ }^{3}$ This control law (that is homogenuous for constant $\mu$ ) has been chosen due to its quality of robustness. Note that $\rho=1$ yields in the classical first order SMC and therefore not treated in [16]. However, it will be treated in this paper since it is relevant to the application in section 5.

${ }^{4}$ The choice of the variation law of $\mu$ is arbitrary. The rules must be fulfilled are to guarantee a sliding mode behaviour when closed-loop system trajectories are far from the desired ones, and close to a linear behaviour when system trajectories are close from the desired ones.
} 
Controller behavior: Let $\mathcal{D}$ be the region around the origin where the variation of $\mu$ occurs, i.e. (see Figure 3) ${ }^{5}$

$$
\mathcal{D}=\left\{\left(z_{1}, \cdots, z_{\rho}\right) \in \mathcal{Z} \mid-\beta \sum_{i=1}^{\rho} \frac{\left|z_{i}\right|}{\left|z_{i}\right|+\varepsilon_{z_{i}}}+1>0\right\}
$$

Suppose that the trajectory of (14) does not evolve in $\mathcal{D}$; then $\mu=0$ and either the classical SMC or controller [17] is applied (depending on the value of $\rho$ ). Given their finite time convergence property and supposing $Q$ as the initial coordinates, the trajectory reaches $\mathcal{D}$ in finite time (see $\widehat{Q R}$ in Figure 3). Once the trajectory is inside $\mathcal{D}$, the variation of $\mu$ takes effect following (16) decreasing the energy consumption, by a control effort point of view, and the chattering effect. Due to the loss of robustness to perturbation/uncertainties $(\mu \neq 0)$, the trajectory might leave $\mathcal{D}$ (see $\widehat{\mathrm{RS}}$ in Figure 3). Hence following (16), $\mu=0$ forcing the trajectory back to $\mathcal{D}$ (see $\widehat{\mathrm{ST}}$ in Figure 3) and so on. In fact, after the system trajectory converges to $\mathcal{D}$ for the first time, it evolves in a domain $\mathcal{D}^{\prime}$ (see $\mathcal{D}^{\prime}$ in Figure 3 ) that is slightly larger than $\mathcal{D} . \varepsilon_{z i}(i \in\{1, \cdots, \rho\}$ ) and $\beta$ influence the size of $\mathcal{D}$ and subsequently $\mathcal{D}^{\prime}$; hence, they influence the global accuracy of the controller. Note that the explicit expression of $\mathcal{D}^{\prime}$ is given in sequel for $\rho=1$ and $\rho=2$.

As previously mentioned, an important property of the proposed control strategy is the reduction of the energy consumption versus the standard SMC or controller [17]. Therefore, one should tune $\varepsilon_{z_{i}}(i=\{1, \cdots, \rho\})$ and $\beta$ in a way to make sure that when $\left|\sigma_{\rho}\right| \geq 1, \mu$ is equal to 0 which is ensured by introducing the following condition on the parameters of $\mu$ in $(16):^{6}$

$$
\sigma_{\rho}\left(\frac{\varepsilon_{z_{1}}}{\beta-1}, \cdots, \frac{\varepsilon_{z \rho}}{\beta-1}\right)<1 .
$$

This discussion is formalized in the following theorem.

Theorem 1 [16]: Consider system (14) under assumptions A1-A3 with $\rho \geq 2$ and controlled by (15)-(16). If $\left(k_{1}, \cdots, k_{\rho}\right)$ are tuned following (18), then there exist $\rho$ positive parameters $\varepsilon_{z_{i}},(i=\{1, \cdots, \rho\})$ and $\beta$ satisfying (19) with $\beta>1$ such that the trajectories of system (14) converge to a vicinity of the origin in a finite time.

Note that this result is given for $\rho=1$ in the sequel and the explicit expression of $\mathcal{D}^{\prime}$ for $\rho=1$ and $\rho=2$ is stated.

\subsection{Main results}

This section formally provides the convergence domain of the system trajectories for both cases $\rho=1$ and $\rho=2$.

Theorem 2: Consider system (14) under assumptions A1-A3 with $\rho=1$ and controlled by (15)(16). If $k_{1}$ fulfills (17), then there exist $\varepsilon_{z_{1}}>0$ and $\beta>1$ satisfying (19) such that the trajectory of system (14) converges, in a finite time, to

$$
\left|z_{1}\right| \leq \frac{\varepsilon_{z_{1}}}{\beta-1} .
$$

\footnotetext{
${ }^{5}$ For clarity and illustration purposes and without loss of generality, Figure 3 is given for $\rho=2$. The logic can be generalizable for $\forall \rho \geq 1$.

${ }^{6}$ With abuse of notation, $\sigma_{\rho}\left(\frac{\varepsilon_{z_{1}}}{\beta-1}, \cdots, \frac{\varepsilon_{z \rho}}{\beta-1}\right)$ is the value of $\sigma_{\rho}$ for $z=\left(\frac{\varepsilon_{z_{1}}}{\beta-1}, \cdots, \frac{\varepsilon_{z \rho}}{\beta-1}\right)$. 


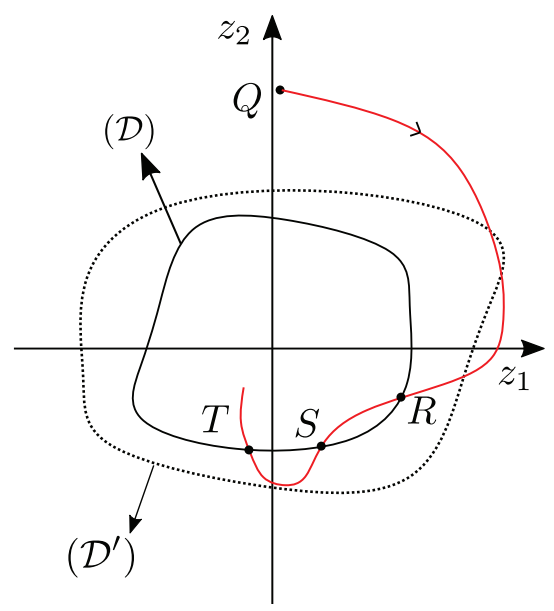

Figure 3: Description of the system trajectory

Proof. Consider the case such that $x \notin \mathcal{D}=\left\{z_{1} \in \mathcal{Z}|| z_{1} \mid<-\beta \frac{\left|z_{1}\right|}{\left|z_{1}\right|+\varepsilon_{z_{1}}}+1\right\}$ i.e. when $z_{1}$ is such that

$$
\left|z_{1}\right|>\frac{\varepsilon_{z_{1}}}{\beta-1} \text {. }
$$

Therefore, according to (16), $\mu=0$ and a first order SMC is applied. Hence, thanks to the features of SMC, the trajectory of the system converges in finite time to $\mathcal{D}$.

Once the system trajectory has reached $\mathcal{D}$, the variation starts, $\mu$ begins evolving between 0 and 1 following (16) and the system trajectories evolve in $\mathcal{D}$. Suppose that the trajectories reach the boundary of $\mathcal{D}$, i.e. $\left|z_{1}\right|=\frac{\varepsilon_{z_{1}}}{\beta-1}$ : in this case, from $(16), \mu=0$. Then, $\dot{z}_{1}$-dynamics read as

$$
\dot{z}_{1}=a(x, t)-b(x, t) k_{1} \operatorname{sign}\left(z_{1}\right) .
$$

As $k_{1}$ is tuned as (17), one has $\dot{z}_{1} \operatorname{sign}\left(z_{1}\right)<0 \forall a(x, t), b(x, t)$ satisfying A3. Thus, as long as $\mu=0$ and the trajectories reach the boundary of $\mathcal{D},\left|z_{1}\right|$ decreases; then, the trajectory is kept in $\mathcal{D}$.

Theorem 3: Consider system (14) under assumptions A1-A3 with $\rho=2$ and controlled by (15)(16). If $k_{1}$ and $k_{2}$ fulfill (18), then there exist $\varepsilon_{z_{1}}>0, \varepsilon_{z_{2}}>0$ and $\beta>1$ satisfying (19) such that the trajectory of system (14) converges, in a finite time, to

$$
\begin{aligned}
& \left|z_{1}\right|<\max \left(\frac{\varepsilon_{z_{1}}}{\beta-1}, \frac{\varepsilon_{z_{2}}^{2}}{2(\beta-1)^{2} K^{*}}\right) \\
& \left|z_{2}\right|<\max \left(\sqrt{\frac{2 K^{*} k_{1}^{2} \varepsilon_{z_{1}}}{\left(k_{1}^{2}+2 K^{*}\right)(\beta-1)}}, \frac{\varepsilon_{z_{2}}}{\beta-1}\right)
\end{aligned}
$$

with $K^{*}=b_{m} k_{2}-a_{M}$.

The proof of this theorem is given in Appendix A. 


\subsection{Application to TWT Control}

The controller proposed in the previous section is applied to the TWT whose nonlinear model is given by (1). The output vector is defined as

$$
y=\left[\begin{array}{c}
y_{\psi} \\
y_{\Omega_{1}} \\
y_{i_{d 1}} \\
y_{\Omega_{2}} \\
y_{i_{d 2}}
\end{array}\right]=\left[\begin{array}{c}
\psi-\alpha \\
\Omega_{1}-\Omega^{*} \\
i_{d 1} \\
\Omega_{2}-\Omega^{*} \\
i_{d 2}
\end{array}\right] .
$$

Note that each element of $y$ corresponds to a sliding variable $s$, as defined in (11). Recall that the control objective is to force $y$ to a vicinity of 0 . The relative degree of $y_{\psi}$ with respect to system (1) is equal to 3 given that

$$
y_{\psi}^{(3)}=\Theta_{1}(x, t)+\Lambda_{1}(x, t) \cdot \Delta \beta
$$

The relative degree of $y_{\Omega_{1}}$ and $y_{\Omega_{2}}$ with respect to (1) is 2 given that

$$
\begin{aligned}
& \ddot{y}_{\Omega_{1}}=\Theta_{2}(x, t)+\Lambda_{2,1}(x, t) \cdot V_{d 1}+\Lambda_{2,2}(x, t) \cdot V_{q 1} \\
& \ddot{y}_{\Omega_{2}}=\Theta_{4}(x, t)+\Lambda_{4,1}(x, t) \cdot V_{d 2}+\Lambda_{4,2}(x, t) \cdot V_{q 2}
\end{aligned}
$$

whereas the relative degree of $y_{i_{d 1}}$ and $y_{i_{d 2}}$ with respect to (1) is 1 given that

$$
\begin{aligned}
& \dot{y}_{i_{d 1}}=\Theta_{3}(x, t)+\Lambda_{3}(x, t) \cdot V_{d 1} \\
& \dot{y}_{i_{d 2}}=\Theta_{5}(x, t)+\Lambda_{5}(x, t) \cdot V_{d 2}
\end{aligned}
$$

Remark 2. Given the relative degree vector of $y$ and the dimension of state space, it gives an internal dynamics that can be defined as $z_{10}=\beta_{1}+\beta_{2}$. Indeed, the following state transformation

$$
\left[\begin{array}{c}
z_{1} \\
z_{2} \\
z_{3} \\
z_{4} \\
z_{5} \\
z_{6} \\
z_{7} \\
z_{8} \\
z_{9} \\
z_{10}
\end{array}\right]=\left[\begin{array}{c}
y_{\psi} \\
\dot{y}_{\psi} \\
\ddot{y}_{\psi} \\
y_{\Omega_{1}} \\
\dot{y}_{\Omega_{1}} \\
y_{\Omega_{2}} \\
\dot{y}_{\Omega_{2}} \\
y_{i_{d_{1}}} \\
y_{i_{d_{2}}} \\
\beta_{1}+\beta_{2}
\end{array}\right]:=\phi(x)
$$

is a diffeomorphism and transforms the nonlinear system into a canonical form. Then, the internal dynamics reads as $\left(T_{\beta}>0\right)$

$$
\dot{z}_{10}=\frac{-z_{10}}{T_{\beta}}+\frac{2 \beta^{o p t}}{T_{\beta}}
$$

This dynamics is totally independent of the other state components and is input-to-state stable (ISS) with respect to the input $\beta^{\text {opt }}$, then the zero dynamics converges exponentially to $2 \beta^{o p t}$. It is the only value injected in the other state components (no peaking phenomena). 
Then, the system can be written as

$$
\left[\begin{array}{l}
y_{\psi}^{(3)} \\
\ddot{y}_{\Omega_{1}} \\
\dot{y}_{i_{d 1}} \\
\ddot{y}_{\Omega_{2}} \\
\dot{y}_{i_{d 2}}
\end{array}\right]=\Theta(x, t)+\Lambda(x, t) \cdot \bar{u}
$$

where

$$
\Theta(x, t)=\left[\begin{array}{c}
\Theta_{1}(x, t) \\
\Theta_{2}(x, t) \\
\Theta_{3}(x, t) \\
\Theta_{4}(x, t) \\
\Theta_{5}(x, t)
\end{array}\right] \text { and } \Lambda(x, t)=\left[\begin{array}{ccccc}
\Lambda_{1}(x, t) & 0 & 0 & 0 & 0 \\
0 & \Lambda_{2,1}(x, t) & \Lambda_{2,2}(x, t) & 0 & 0 \\
0 & \Lambda_{3}(x, t) & 0 & 0 & 0 \\
0 & 0 & 0 & \Lambda_{4,1}(x, t) & \Lambda_{4,2}(x, t) \\
0 & 0 & 0 & 0 & \Lambda_{5}(x, t)
\end{array}\right]
$$

the expressions of $\Theta(x, t)$ and $\Lambda(x, t)$ being given in Appendix $\mathrm{B}$.

The parameters on which uncertainties have been considered are the inductance $L_{d}$, the stator resistance $R_{s}$ and the drag force coefficients $C_{d, i}$. Therefore, each of these parameters is divided to a nominal and an uncertain part. Subsequently, $\Theta(x, t)$ and $\Lambda(x, t)$ can be written as follows

$$
\Theta(x, t)=\bar{\Theta}(x, t)+\Delta \Theta(x, t), \quad \Lambda(x, t)=\bar{\Lambda}(x, t)+\Delta \Lambda(x, t)
$$

where $\bar{\Theta}(x, t)$ and $\bar{\Lambda}(x, t)$ are the nominal parts of $\Theta(x, t)$ and $\Lambda(x, t)$, respectively, and $\Delta \Theta(x, t)$ and $\Delta \Lambda(x, t)$ are the uncertain parts, respectively.

Define the control input $\bar{u}$ as

$$
\bar{u}=[\bar{\Lambda}(x, t)]^{-1}[-\bar{\Theta}(x, t)+\vartheta]
$$

which gives

$$
\left[\begin{array}{l}
y_{\psi}^{(3)} \\
\ddot{y}_{\Omega_{1}} \\
\dot{y}_{i_{d 1}} \\
\ddot{y}_{\Omega_{2}} \\
\dot{y}_{i_{d 2}}
\end{array}\right]=\underbrace{\left(\Delta \Theta(x, t)-\Delta(x, t) \bar{\Lambda}(x, t)^{-1} \bar{\Theta}(x, t)\right)}_{\varphi_{1}}+\underbrace{\left(I_{5 \times 5}+\Delta \Lambda(x, t) \bar{\Lambda}(x, t)^{-1}\right)}_{\varphi_{2}} \vartheta
$$

with $\vartheta$, the "new" control defined in the sequel. The matrix $\Lambda(x, t)$ and its nominal value $\Lambda_{N}(x, t)$ can be inverted if

$$
\psi-\alpha \neq( \pm 2 \varrho+1) \frac{\pi}{2}
$$

where $\varrho \in \mathbb{N}$. If $\psi-\alpha=( \pm 2 \varrho+1) \frac{\pi}{2}$, the arm linking the wind turbines is strictly parallel to the direction of the wind: no drag force can be created. Hence, one considers that condition (34) is satisfied.

A4. The parametric uncertainties have limites magnitudes with respect to their nominal value, and there is no lose of structural properties.

Assumption A4 implies that $\varphi_{2}$ has eigenvalues close to zero: as a consequence, the matrix $I_{5 \times 5}$ 
is a dominant matrix with respect to $\varphi_{2}$. Moreover, the term $\varphi_{1}$ can be considered as a matching perturbation.

As shown previously, the zer-dynamics is stable. Supposing that initial conditions are bounded (that is practically reasonable), and given Remark $1, \varphi_{1}$ and $\varphi_{2}$ are bounded satisfying Assumption 3. Hence, control law (32) yields in almost decoupled dynamics of the MIMO system (30). Then, the "new" control input, $\vartheta$, is given as follows

$$
\vartheta=\left[\begin{array}{c}
-k_{\rho, \psi}\left\lceil\sigma_{\rho, \psi}\right\rfloor^{\mu_{\psi}} \\
-k_{\rho, \Omega 1}\left\lceil\sigma_{\rho, \Omega_{1}}\right\rfloor^{\mu_{\Omega_{1}}} \\
-k_{\rho, i_{d 1}}\left\lceil\sigma_{\rho, i_{d 1}}\right\rfloor_{i_{d 1}}^{\mu_{i_{d 1}}} \\
-k_{\rho, \Omega 2}\left\lceil\sigma_{\rho, \Omega_{2}} \mu_{\Omega_{2}}^{\mu_{\Omega_{2}}}\right. \\
-k_{\rho, i_{d 2}}\left\lceil\sigma_{\rho, i_{d 2}}\right\rfloor^{\mu_{i_{d 2}}}
\end{array}\right] .
$$

Accordingly with the output relative degree

- $\sigma_{\rho, \psi}$ and $\mu_{\psi}$ are calculated following (15) and (16) respectively for $\rho=3$ and $z_{1}=y_{\psi}$.

- $\sigma_{\rho, \Omega_{1}}$ and $\mu_{\Omega_{1}}$ (respectively $\sigma_{\rho, \Omega_{2}}$ and $\mu_{\Omega_{2}}$ ) are calculated for $\rho=2$ and $z_{1}=y_{\Omega_{1}}$ (respectively $z_{1}=y_{\Omega_{2}}$ ).

- $\sigma_{\rho, i_{d 1}}$ and $\mu_{i_{d 1}}$ (respectively $\sigma_{\rho, i_{d 2}}$ and $\mu_{i_{d 2}}$ ) are calculated for $\rho=1$ and $z_{1}=y_{i_{d 1}}$ (respectively $z_{1}=y_{i_{d 2}}$ ).

By setting the gains $K_{\rho, \star}$ with $\star=\left\{\psi, \Omega_{1}, \Omega_{2}, i_{d 1}, i_{d 2}\right\}$ such that conditions (17) $(\rho=1)$ and (18) $(\rho=\{2,3\})$ are satisfied, the finite time convergence to a vicinity of the origin of the output vector $y$ is ensured. The parameter tuning will be detailed in the next subsection.

\section{Simulation results}

The proposed controller is implemented on the twin wind turbine structure. The main parameters of the twin wind turbines [21] can be found in Table 10 in [5]. $\beta^{\text {opt }}$ and $\lambda^{\text {opt }}$ are set at $2^{\circ}$ and 7.3 respectively. Simulations have been performed using MATLAB/Simulink with sampling period $T_{e}=0.1 \mathrm{~ms}$. A filter of the form $\frac{s}{\tau s+1}$ is used to estimate the first and second order time derivatives of $\psi$ and the first order time derivative of $\Omega$. The performances of the proposed controller are compared to controller [17] by evaluating the mean generated power and the oscillations of the pitch angles and electromagnetic torques. The indicator used to evaluate the oscillations is the function Var defined as

$$
\operatorname{Var}_{\left[\zeta_{1}, \zeta_{2}\right]}(h)=\sum_{i=0}^{N-1}\left|h\left(t_{i+1}\right)-h\left(t_{i}\right)\right|
$$

where $h$ is a real valued function and the set of instants $\left\{t_{0}, t_{1}, \cdots, t_{N}\right\}$ is a partition of $\left[\zeta_{1}, \zeta_{2}\right]$. Given that the simulation results of both wind turbines are similar, only the ones of wind turbine 1 will be displayed. The wind speed is modeled as

$$
V=\left(V_{m}+V_{d}\right) \cdot \cos (\psi-\alpha)
$$

with $V_{m}$ the average wind speed taken equal to $10 \mathrm{~m} / \mathrm{s}$, and $V_{d}$ a time-varying term defined as a white noise. In order to test the robustness of the controllers to parametric uncertainties, a $20 \%$ variation with respect to their nominal value is taken on $R_{s}$ and $L_{d}$ and a $50 \%$-variation is taken 
on $C_{d i}$. The proposed controller parameters are tuned as given in Table 2. Recall that controller [17] has the same structure as the proposed controller but with $\mu=0, \forall t>0$. Hence, the latter's gains are taken equal to those of the proposed controller (see gain $k_{\rho}$ in Table 2) for an effective performance comparison between the two.

\begin{tabular}{l||c|c|c|c|c} 
Controlled variable & Gain $k_{\rho, \star}$ & $\beta$ & $\varepsilon_{z_{1}}$ & $\varepsilon_{z_{2}}$ & $\varepsilon_{z_{3}}$ \\
\hline Yaw angle $\psi(\rho=3)$ & 2.2 & 1.1 & $5 \cdot 10^{-5}$ & $10^{-3}$ & $10^{-1}$ \\
Velocities $\Omega_{1}$ and $\Omega_{2}(\rho=2)$ & 100 & 11 & $2 \cdot 10^{-1}$ & 5 & - \\
Direct currents $(\rho=1)$ & 10 & 11 & $3 \cdot 10^{-3}$ & - & -
\end{tabular}

Table 2: Proposed controller parameters.

The wind direction $\alpha$ changes between $10^{\circ}$ and $-10^{\circ}$ (Figure 4 - Top). When the direction of the wind changes, the pitch angles $\beta_{1}$ and $\beta_{2}$ are actuated (Figure 4 - Bottom) and a drag force difference is generated which in its turn leads to the rotation of the whole structure (Figure 4 Top).

Initially the value of $\mu_{\psi}$ is equal to zero (see Figure 5 - Left) this is because the yaw angle $\psi$ does not track the wind direction $\alpha$; hence, $\mu_{\psi}=0$ forcing $\psi$ to $\alpha$. When the latter is achieved and a steady state is attained $(10<t<35 \mathrm{sec})$, then $\mu$ starts varying between 0 and 1 reducing the chattering phenomena. A similar logic can be applied to $\mu_{\Omega_{1}}$ (see Figure 5 - Right) and $\mu_{i_{d 1}}$ (in fact in this case $\mu_{i_{d 1}}=1$ almost all the time meaning that the direct currents are not affected by the uncertainties considered in this simulation).

The reduction in the chattering in the steady state caused by the variation of $\mu(\mu \neq 0)$ is manifested by the reduction of the oscillations of the electromagnetic torque $\Gamma_{e m 1}$ and that of the pitch angle $\beta_{1}$ (Figure 6 - Middle, see Figure 4 - Middle, and $\operatorname{Var}\left(\Gamma_{e m 1}\right)$ and $\operatorname{Var}\left(\beta_{1}\right)$ in Table 6) all while keeping good yaw tracking and optimal power production. This fact is important in decreasing the fatigue loads hence increasing the lifetime of the structure. Notice that the current $i_{d 1}$ (see Figure 6-Bottom) with the proposed controller is smoother than with controller [17] (which is the first order sliding mode controller used for the control of the TWT in [5]) with a better accuracy tracking since chattering is reduced.

Notice that controller [17] has a faster convergence to the required yaw angle than the proposed controller (see Figure 4 - Top $43<t<45 \mathrm{sec}$ ); however it does not affect the mean power produced by the system (see power generated in Figure 6 and mean power in Table 3). 

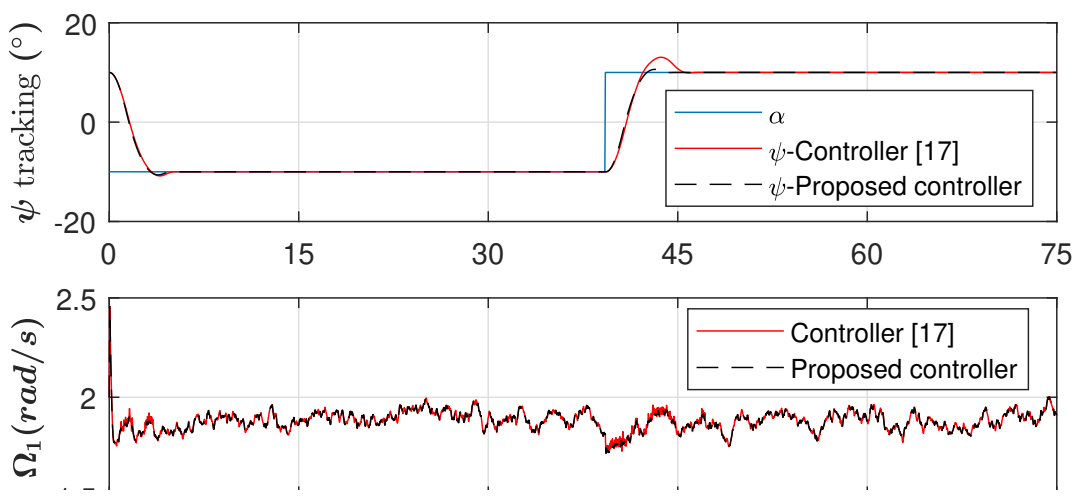

Figure 4:

Middle -

(sec).

Turbine 1
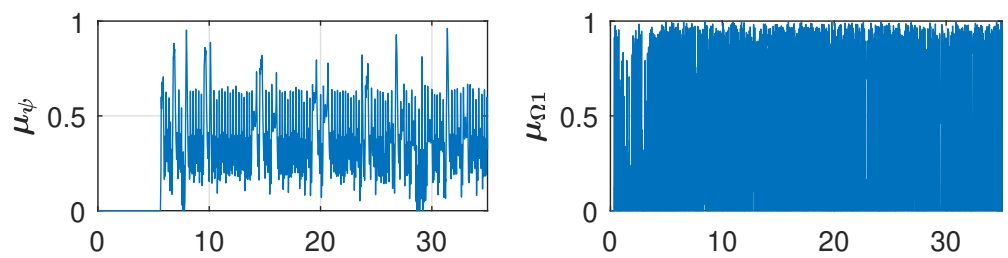

Figure 5: Left $-\mu_{\psi}$ versus time (sec). Right $-\mu_{\Omega_{1}}$ versus time (sec). 

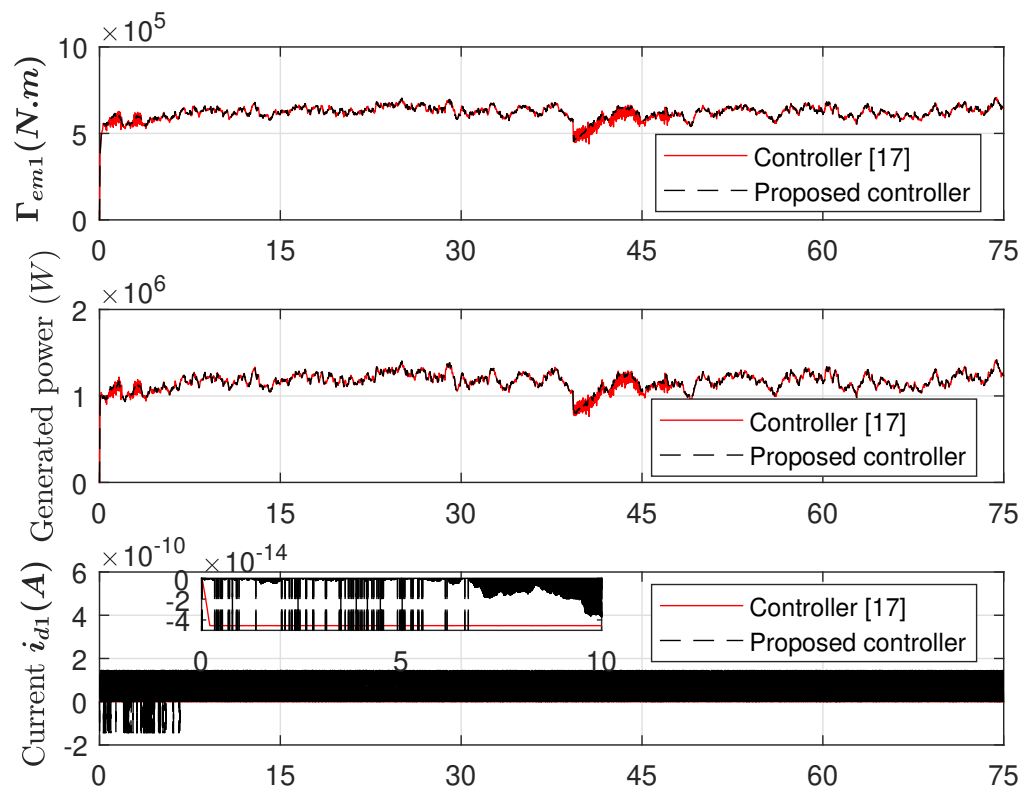

Figure 6: Comparison controller [17] and the proposed controller - Top - Electromagnetic torque $\Gamma_{e m 1}(N . m)$ versus time $(\mathrm{sec})$. Bottom - Generated power for Wind Turbine $1(\mathrm{~W})$ versus time $(\mathrm{sec})$.

\begin{tabular}{|l|c|c|}
\cline { 2 - 3 } \multicolumn{1}{c|}{} & Controller [17] & Proposed controller \\
\hline $\operatorname{Var}\left(\Gamma_{e m 1}\right)(N \cdot m)$ & $9.487 \cdot 10^{6}$ & $7.800 \cdot 10^{6}$ \\
\hline $\operatorname{Var}\left(\beta_{1}\right)\left({ }^{\circ}\right)$ & 75.030 & 18.994 \\
\hline Mean power $(W)$ & $1.153 \cdot 10^{6}$ & $1.151 \cdot 10^{6}$ \\
\hline
\end{tabular}

Table 3: Comparison controller [17] and proposed controller $-\operatorname{Var}\left(\Gamma_{e m 1}\right)(N \cdot m)$ and $\operatorname{Var}\left(\beta_{1}\right)\left({ }^{\circ}\right)$ in the steady state $(10<t<35 \mathrm{sec})$ and mean power.

\section{Conclusion}

The domain of convergence of a new control methodology has been given for input-output relative degree equal to 1 and 2 . The proposed controller ensures high accuracy tracking while decreasing the control effort and the chattering. The effectiveness of the new methodology for the control of a TWT is shown. The proposed control law ensures power output maximization, with reduced electromagnetic torque and pitch angle oscillations, resulting in the improvement of the structure lifetime. Future works will be dedicated to study the closed-loop stability of the proposed controller coupled with differentiators as well as applying this control strategy to floating wind systems that have an increased implementation complexity.

\section{References}

[1] V. I. Utkin, Sliding modes in control and optimization. Springer-Verlag Berlin Heidelberg, 1992. 
[2] Y. Shtessel, C. Edwards, L. Fridman, and A. Levant, Sliding mode control and observation. Springer, 2014.

[3] K. Jezernik, J. Korelič, and R. Horvat, "Pmsm sliding mode fpga-based control for torque ripple reduction," IEEE Transactions on Power Electronics, vol. 28, no. 7, pp. 3549-3556, 2013.

[4] C. Edwards and Y. B. Shtessel, "Adaptive continuous higher order sliding mode control," Automatica, vol. 65, pp. 183-190, 2016.

[5] I. Guenoune, F. Plestan, A. Chermitti, and C. Evangelista, "Modeling and robust control of a twin wind turbines structure," Control Engineering Practice, vol. 69, pp. 23-35, 2017.

[6] A. Levant, "Universal single-input-single-output (siso) sliding-mode controllers with finite-time convergence," IEEE transactions on Automatic Control, vol. 46, no. 9, pp. 1447-1451, 2001.

[7] A. Levant, "Homogeneity approach to high-order sliding mode design," Automatica, vol. 41, no. 5, pp. 823-830, 2005.

[8] E. Bernuau, D. Efimov, W. Perruquetti, and A. Polyakov, "On homogeneity and its application in sliding mode control," Journal of the Franklin Institute, vol. 351, no. 4, pp. 1866-1901, 2014.

[9] Y. V. Orlov, Discontinuous systems: Lyapunov analysis and robust synthesis under uncertainty conditions. Springer Science \& Business Media, 2008.

[10] A. Polyakov and A. Poznyak, "Lyapunov function design for finite-time convergence analysis:"twisting" controller for second-order sliding mode realization," Automatica, vol. 45, no. 2, pp. 444-448, 2009.

[11] A. Polyakov and A. Poznyak, "Unified lyapunov function for a finite-time stability analysis of relay second-order sliding mode control systems," IMA Journal of Mathematical Control and Information, vol. 29, no. 4, pp. 529-550, 2012.

[12] E. Tahoumi, M. Ghanes, F. Plestan, and J.-P. Barbot, "A new controller switching between linear and twisting algorithms," in 2018 Annual American Control Conference (ACC), pp. 6150-6155, IEEE, 2018.

[13] E. Tahoumi, F. Plestan, M. Ghanes, and J.-P. Barbot, "Adaptive exponent parameter: a robust control solution balancing between linear and twisting controllers," in 2018 15th International Workshop on Variable Structure Systems (VSS), pp. 186-191, IEEE, 2018.

[14] A. Levant, "Sliding order and sliding accuracy in sliding mode control," International journal of control, vol. 58 , no. 6, pp. 1247-1263, 1993.

[15] E. Tahoumi, F. Plestan, M. Ghanes, and J.-P. Barbot, "A controller switching between twisting and linear algorithms for an electropneumatic actuator," in 2018 European Control Conference (ECC), pp. 2368-2373, IEEE, 2018.

[16] E. Tahoumi, F. Plestan, M. Ghanes, and J.-P. Barbot, "Robust and energy efficient control schemes based on higher order sliding mode," in 2019 European Control Conference (ECC), IEEE, 2019.

[17] E. Cruz-Zavala and J. Moreno, Lyapunov approach to higher-order sliding mode design, pp. 3-28. Recent trends in sliding mode control edited by L. Fridman, J.-P. Barbot and F. Plestan. The Institution of Engineering and Technology, 2016

[18] A. Herskovits, O. Laffitte, P. Thome, and A. Tobie, "V-shaped, bi-rotor wind generator on a spar floating structure," French Patent WO2014060420 A, vol. 1, p. 2014, 2012.

[19] S. Georg, H. Schulte, and H. Aschemann, "Control-oriented modelling of wind turbines using a takagi-sugeno model structure," in 2012 IEEE International Conference on Fuzzy Systems, pp. 1-8, IEEE, 2012.

[20] H. Shariatpanah, R. Fadaeinedjad, and M. Rashidinejad, "A new model for PMSG-based wind turbine with yaw control," IEEE transactions on energy conversion, vol. 28, no. 4, pp. 929-937, 2013.

[21] A. Uehara, A. Pratap, T. Goya, T. Senjyu, A. Yona, N. Urasaki, and T. Funabashi, "A coordinated control method to smooth wind power fluctuations of a PMSG-based wecs," IEEE Transactions on energy conversion, vol. 26, no. 2, pp. 550-558, 2011.

[22] A. Levant, "Principles of 2-sliding mode design," Automatica, vol. 43, no. 4, pp. 576-586, 2007. 


\section{Appendix A: Proof of Theorem 3}

When the relative degree of the system is equal to 2, i.e. $\rho=2$, the controller from [17] is discontinuous on the same curve in the phase plan as the controller from [7] $\left(u=-k_{2}\left\lceil z_{2}+k_{1}\left\lceil z_{1}\right\rfloor^{\frac{1}{2}}\right\rfloor^{0}\right)$ described as $z_{2}=-k_{1}\left\lceil z_{1}\right\rfloor^{\frac{1}{2}}$ (see $\mathcal{L}$ in Figure 7). Hence, following [22], if the gains are tuned as (18), the system trajectory inevitably hits the manifold $\mathcal{L}$ and then will slide on it until the origin is reached. This fact is essential in the determination of the domain of convergence of the trajectory when controller (15)-(16) is applied to system (14) when $\rho=2$ which will be detailed in the following.

By a general point-of-view, the trajectory of the system is outside $\mathcal{D}$ : therefore, $\mu=0$ and the controller from [17] is applied. As previously mentioned, the system trajectory will hit $\mathcal{L}$ and slide on it; therefore, it will reach $\mathcal{D}$ in a finite time. However, due to the presence of perturbations and uncertainties, the trajectory will potentially leave $\mathcal{D}$ from

Case 1. $\widehat{A B}($ resp. $\widehat{C D})$

Case 2. $\widehat{B E}($ resp. $\overparen{D F})$

The trajectory cannot leave $\mathcal{D}$ through $\overparen{E C}$ since at $\overparen{E C}, \mu=0$ meaning that $\dot{z}_{2}>0$ and $z_{2}<0$; therefore, $z_{2}$ cannot decrease and $z_{1}$ cannot increase. Similarly, the trajectory cannot leave $\mathcal{D}$ through $\widehat{F A}$.

The domain of convergence for each case will be presented in the sequel.

Case 1: Suppose that the trajectory leaves $\mathcal{D}$ through $\overparen{A B}$ at a point $M$ (see $M$ in Figure 7a). Considering the worst case, $\dot{z}_{2}=-K^{*}=-b_{m} k_{2}+a_{M}$ gives the most external trajectory from the origin. Therefore, the expression of $\widehat{M N}$ is ${ }^{7}$

$$
z_{1}=\frac{-z_{2}^{2}+z_{2}^{2}(M)}{2 K^{*}}+z_{1}(M)
$$

From (16) and for $\rho=2$ one has that $\alpha=0$ when

$$
\left|z_{1}\right| \geq \frac{\varepsilon_{z_{1}} \varepsilon_{z_{2}}-(\beta-1) \varepsilon_{z_{2}} z_{2}(M)}{(2 \beta-1) z_{2}(M)+\varepsilon_{z_{2}}(\beta-1)}
$$

then, the expression of $z_{1}(M)$ is

$$
z_{1}(M)=\frac{\varepsilon_{z_{1}} \varepsilon_{z_{2}}-(\beta-1) \varepsilon_{z_{2}} z_{2}(M)}{(2 \beta-1) z_{2}(M)+\varepsilon_{z_{2}}(\beta-1)}
$$

Hence, combining (37) and (39) the expression of $z_{1}(N)$ is deduced:

$$
z_{1}(N)=\frac{z_{2}^{2}(M)}{2 K^{*}}+\frac{\varepsilon_{z_{1}} \varepsilon_{z_{1}}-(\beta-1) \varepsilon_{z_{2}} z_{2}(M)}{(2 \beta-1) z_{2}(M)+\varepsilon_{z_{2}}(\beta-1)} .
$$

\footnotetext{
${ }^{7}$ Denote $z_{1}(M)\left(\right.$ resp. $\left.z_{2}(M)\right)$ as the $z_{1}\left(\right.$ resp. $\left.z_{2}\right)$ coordinate of point $M$.
} 
The maximum of this expression on the interval $z_{2}(M) \in\left[0, \frac{\varepsilon_{z_{2}}}{\beta-1}\right]$ is

$$
\max \left(z_{1}(N)\right)=\max \left(\frac{\varepsilon_{z_{1}}}{\beta-1}, \frac{\varepsilon_{z_{2}}^{2}}{2(\beta-1)^{2} K^{*}}\right)
$$

After the trajectory crosses the $z_{1}$ - axis it will enter again $\mathcal{D}$ through $\widehat{\mathrm{BC}}$ (see $\widehat{\mathrm{NO}}$ in Figure 7a). This is due to the fact that $\widehat{\mathrm{MN}}$ and $\widehat{\mathrm{NO}}$ are symmetric with respect to $z_{1}-$ axis. Note that in case the trajectory hits $\mathcal{L}$ before hitting $\widehat{\mathrm{BC}}$ (see $\widehat{N O}^{\prime}$ in Figure 7a) then it will slide on $\mathcal{L}$ and then enter $\mathcal{D}$ through $\widehat{B C}$ (see $\widehat{\mathrm{O}^{\prime} \mathrm{E}}$ in Figure 7a). This fact, combined with the result from (41) gives that when the trajectory leaves $\mathcal{D}$ from $\widehat{\mathrm{AB}}$ and $\widehat{\mathrm{CD}}$ (due to symmetry) then

$$
\begin{aligned}
& \left|z_{1}\right| \leq \max \left(\frac{\varepsilon_{z_{1}}}{\beta-1}, \frac{\varepsilon_{z_{2}}^{2}}{2(\beta-1)^{2} K^{*}}\right) \\
& \left|z_{2}\right| \leq \frac{\varepsilon_{z_{2}}}{\beta-1}
\end{aligned}
$$

Case 2. When the trajectory leaves $\mathcal{D}$ at a point $H$ on $\widehat{\mathrm{BC}}$ (see $H$ in Figure $7 \mathrm{~b}$ ), $\mu=0$; then, the controller from [17] is applied. As previously mentioned, the manifold defined by $\mathcal{L}$ in the phase plan is attractive; therefore, the trajectory will hit $\mathcal{L}$ at point $I$ and start converging towards the origin (see $\widehat{\mathrm{IE}}$ in Figure 7b). Still considering the worst case, $\dot{z}_{2}=-K^{*}$, the expression of $\widehat{\mathrm{HI}}$ is also given by

$$
z_{1}=\frac{-z_{2}^{2}+z_{2}^{2}(H)}{2 K^{*}}+z_{1}(H)
$$

At point $I$ one has:

$$
z_{1}(I)=\frac{1}{k_{1}^{2}} z_{2}^{2}(I)
$$

Hence, by combining (44) and (43) the following expression of $z_{2}(I)$ is obtained:

$$
z_{2}^{2}(I)=\frac{k_{1}^{2}}{k_{1}^{2}+2 K^{*}}\left(z_{2}^{2}(H)+2 K^{*} \frac{\varepsilon_{z_{1}} \varepsilon_{z_{2}}+(\beta-1) z_{2}(H) \varepsilon_{z_{1}}}{-(2 \beta-1) z_{2}(H)+(\beta-1) \varepsilon_{z_{2}}}\right)
$$

The maximum of the latter is obtained when $z_{2}(H)=0$

$$
z_{2}(I)=-\sqrt{\frac{2 K^{*} k_{1}^{2} \varepsilon_{z_{1}}}{\left(k_{1}^{2}+2 K^{*}\right)(\beta-1)}}
$$

or when $H$ coincides with point $E$ which is the intersection between $\mathcal{L}$ and $\mathcal{D}$ (see $E$ in Figure $7 b)$. Considering the worst case, that is, when $z_{2}(E)$ maximal in absolute value and therefore coinciding with point $\mathrm{C}$ (i.e. $k_{1} \rightarrow \infty$ and therefore $z_{2}(H)=-\frac{\varepsilon_{z_{2}}}{\beta-1}$ )

$$
z_{2}(I)=-\frac{\varepsilon_{z_{2}}}{\beta-1} \sqrt{\frac{k_{1}^{2}}{k_{1}^{2}+2 K^{*}} .}
$$

Note that the latter expression gives a boundary for $z_{2}$ smaller than that in (42) and therefore will be discarded when calculating the ultimate convergence domain. In addition to that, with 
$k_{1} \rightarrow \infty$ one has the same convergence domain as in (42). However, one states that if the system trajectory leaves from $\widehat{\mathrm{BE}}$ and $\widehat{\mathrm{AF}}$ (due to symmetry), the convergence boundary is

$$
\begin{aligned}
& \left|z_{1}\right|<\frac{\varepsilon_{z_{1}}}{\beta-1} \\
& \left|z_{2}\right|<\max \left(\sqrt{\frac{2 K^{*} k_{1}^{2} \varepsilon_{z_{1}}}{\left(k_{1}^{2}+2 K^{*}\right)(\beta-1)}}, \frac{\varepsilon_{z_{2}}}{\beta-1} \sqrt{\frac{k_{1}^{2}}{k_{1}^{2}+2 K^{*}}}\right)
\end{aligned}
$$

As a conclusion, by combining the 2 previous cases, the ultimate convergence boundary of the system is

$$
\begin{aligned}
& \left|z_{1}\right|<\max \left(\frac{\varepsilon_{z_{1}}}{\beta-1}, \frac{\varepsilon_{z_{2}}^{2}}{2(\beta-1)^{2} K^{*}}\right) \\
& \left|z_{2}\right|<\max \left(\sqrt{\frac{2 K^{*} k_{1}^{2} \varepsilon_{z_{1}}}{\left(k_{1}^{2}+2 K^{*}\right)(\beta-1)}}, \frac{\varepsilon_{z_{2}}}{\beta-1}\right)
\end{aligned}
$$

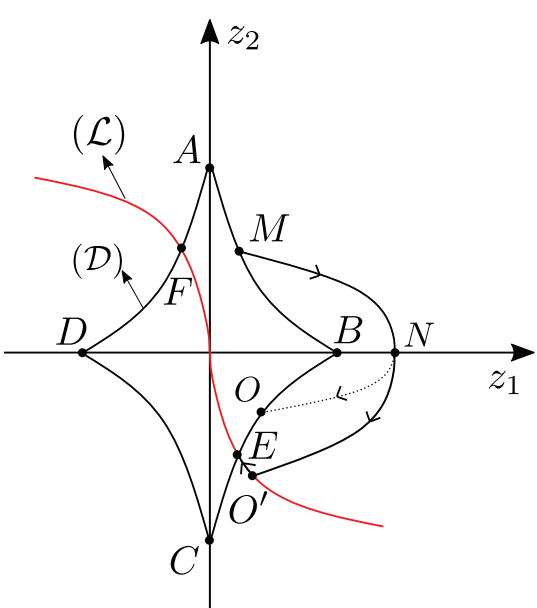

(a) Case 1

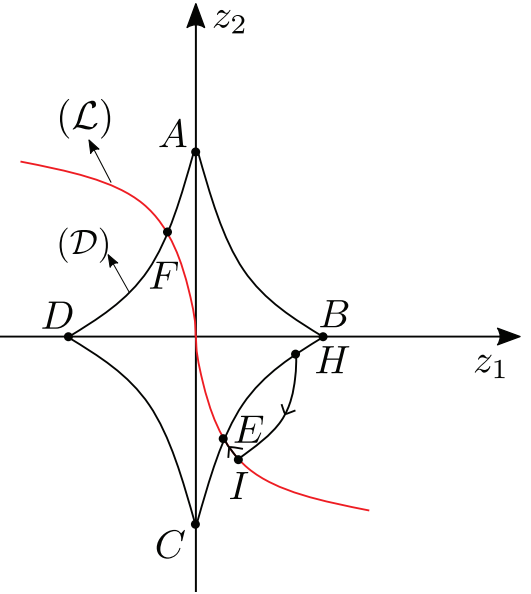

(b) Case 2

Figure 7: Description of the system trajectory in the phase plan $\left(z_{1}, z_{2}\right)$. 


\section{Appendix B}

The expressions of $\Theta(x, t)$ and $\Lambda(x, t)$ are given

$$
\Theta(x, t)=\left[\begin{array}{c}
-\frac{D_{r}}{K_{r}} \ddot{\psi}+\frac{C L}{K_{r} T_{\beta}} \mathcal{B}\left(\beta_{1}-\beta_{2}\right)+\frac{C L}{K_{r}}\left(\beta_{1}-\beta_{2}\right) \dot{\mathcal{B}}+\frac{\mathcal{B} L}{K_{r}}\left(\beta_{1}-\beta_{2}\right) \dot{C} \\
\frac{1}{J} \dot{\Gamma}_{a 1}-\left(f_{1}+f_{2} i_{d 1}\right)\left[-f_{3} i_{q 1}-f_{4} \Omega_{1} i_{d 1}-f_{5} \Omega_{1}\right]-f_{2} i_{q 1}\left[-f_{6} i_{d 1}+f_{7} \Omega_{1} i_{q 1}\right]-\frac{f_{v}}{J} \dot{\Omega}_{1}-\ddot{\Omega}_{1}^{*} \\
\frac{-R_{s}}{L_{d}} i_{d 1}+\frac{P L_{q}}{L_{d}} \Omega_{1} i_{q 1} \\
\frac{1}{J} \dot{\Gamma}_{a 2}-\left(f_{1}+f_{2} i_{d 2}\right)\left[-f_{3} i_{q 2}-f_{4} \Omega_{2} i_{d 2}-f_{5} \Omega_{2}\right]-f_{2} i_{q 2}\left[-f_{6} i_{d 2}+f_{7} \Omega_{2} i_{q 2}\right]-\frac{f_{v}}{J} \dot{\Omega}_{2}-\ddot{\Omega}_{2}^{*} \\
\frac{-R_{s}}{L_{d}} i_{d 2}+\frac{P L_{q}}{L_{d}} \Omega_{2} i_{q 2}
\end{array}\right]
$$

$$
\Lambda(x, t)=\left[\begin{array}{ccccc}
\frac{-2}{K_{r} T_{\beta}} L C \mathcal{B} & 0 & 0 & 0 & 0 \\
0 & \frac{-f_{2}}{L_{d}} i_{q 1} & \frac{-1}{L_{q}}\left(f_{1}+f_{2} i_{d 1}\right) & 0 & 0 \\
0 & \frac{1}{L_{d}} & 0 & 0 & 0 \\
0 & 0 & 0 & \frac{-f_{2}}{L_{d}} i_{q 2} & \frac{-1}{L_{q}}\left(f_{1}+f_{2} i_{d 2}\right) \\
0 & 0 & 0 & \frac{1}{L_{d}} & 0
\end{array}\right]
$$

with $f_{i}, i \in\{1,2, \ldots, 7\}$

$$
f_{1}=\frac{p \phi_{f}}{J}, f_{2}=\frac{p\left(L_{d}-L_{q}\right)}{J}, f_{3}=\frac{R_{s}}{L_{q}}, f_{4}=\frac{p L_{d}}{L_{q}}, f_{5}=\frac{p \phi_{f}}{L_{q}}, f_{6}=\frac{R_{s}}{L_{d}}, f_{7}=\frac{p L_{q}}{L_{d}} .
$$

Note that the expression of $F_{d, 1}-F_{d, 2}$ is given as ${ }^{8}$

$$
F_{d, 1}-F_{d, 2}=C \mathcal{B}\left(\beta_{1}-\beta_{2}\right)
$$

where $C=\frac{1}{2} \rho \pi(V \cos (\psi-\alpha))^{2}$ and $\mathcal{B}=b_{0}+b_{1} \lambda_{i}+b_{2} \lambda_{i}^{2}+b_{3} \lambda_{i}^{3}$ with $b_{0}=-0.008608$, $b_{1}=0.0063, b_{2}=-0.0015$ and $b_{3}=0.000118$.

The time derivatives $\dot{\mathcal{B}}$ and $\dot{C}$ found in $\Theta(x, t)$ read as

$$
\begin{aligned}
\dot{\mathcal{B}} & =\frac{\partial \mathcal{B}}{\partial t}=\frac{\partial \mathcal{B}}{\partial \lambda} \cdot \frac{\partial \lambda}{\partial t}=\left(b_{1}+2 b_{2} \lambda+3 b_{3} \lambda^{2}\right) \cdot \frac{\partial}{\partial t}\left(\frac{\Omega R}{V \cos (\psi-\alpha)}\right) \\
& =\left(b_{1}+2 b_{2} \lambda+3 b_{3} \lambda^{2}\right) \cdot\left(\frac{R}{V \cos (\psi-\alpha)} \dot{\Omega}+\lambda \operatorname{tg}(\psi-\alpha) \dot{\psi}-\frac{\lambda}{V} \dot{V}\right) \\
\dot{C} & =\frac{\partial C}{\partial t}=\frac{\partial C}{\partial V} \cdot \frac{\partial V}{\partial t}+\frac{\partial C}{\partial \psi} \cdot \frac{\partial \psi}{\partial t} \\
& =\frac{2 C}{V} \dot{V}-2 \operatorname{tg}(\psi-\alpha) \dot{\psi}
\end{aligned}
$$

${ }^{8}$ For more details on how this expression is obtained the reader is referred to [5]. 
The filter $\frac{s}{\tau s+1}$ is used to estimate the derivative of $V$. 